Volume: 2, Issue: 1, January-March 2017, Pages: 131, DOI: http://dx.doi.org/10.19082/ah131

\title{
A CLINICAL DECISION SUPPORT SYSTEM FOR PREDICTING HEART DISEASE
}

\author{
Mohammad Reza afrash ${ }^{1}$, Mehdi Khalili ${ }^{2}$, Maral Sedigh Salekde ${ }^{r}$
}

1: M. S. Student in Computer Engineering, Research Institute of Jahad Daneshgahi, Ahvaz, Iran

2: Assistant Professor, Department of Computer and Informatics Engineering, Payame Noor University, Tehran, Iran

3: M. S. Student Department of Computer and Informatics Engineering, Payame Noor University, Tehran, Iran

Correspondence:

Mohammad Reza Afrash. Tel: +989389408230, E-mail: Mmdrza.lp19@gmail.com

\section{TYPE OF ARTICLE: CONFERENCE ABSTRACT}

\begin{abstract}
Introduction: Heart disease is a term that covers a range of disorders that affect heart. Heart disease is the leading cause of death in different countries. Medical diagnosis is an important and complicated task that should be performed properly and efficiently. Unfortunately, some doctors are not sufficiently adept at subspecialty, and in many cases they have few resources. The need for new tools able to help doctors in predicting and diagnosis heart disease is highly recognized.

Methods: In the present study, first the data set containing total instances of 380 with 14 attributes containing eight nominal and six numeric were obtained from the UCI machine learning repository. Second, because our purpose is classification and choice of the most suitable technique based on many evaluation measures (specificity, sensitivity, kappa, $f$-measure, accuracy, and Roc area) for our clinical heart disease prediction system, five data mining techniques were used: the naïve Bayes, artificial neural networks, random forest and C4.5 decision trees and random tree algorithms. In order to develop a computer-based clinical decision support system, the computer programming language employed was the C\#.NET programming language.
\end{abstract}

Results: From the experimental result, it is observed that the random forest algorithm with $(89.73,0.957,0.084$, $0.985,0.8404)$ for accuracy, $f$-measure, specificity, sensitivity, and kappa rate produce a higher performance for our classification model when compared with other algorithms. Finally, the random forest algorithm in this proposed work was implemented using Microsoft C\# programming language.

Conclusion: Heart disease is the main cause of death in different countries. Medical diagnosis is important to save the patient and offer other medical and health care services, but it is a complicated task. In the present study, we develop a computer-based clinical decision support system that can help doctors to predict and diagnose heart disease by using data mining techniques. First, a data set with 14 attributes was utilized. Then, in order to choose the best algorithm for the utilized system, the researchers compared many data mining algorithms such as Naïve Bayes, neural networks, random forest, and C4.5 decision tree and random tree by using several performance assessments. The applied data set in this research was obtained from several medical centers and hospitals from the UCI machine learning repository, containing total instances of 380, with 14 attributes containing eight nominal and six numeric. The results indicated the random forest technique as the best algorithm with an 89.73 classification rate. Finally, random forest algorithm implemented in this proposed work was analyzed by Microsoft C\# programming language.

KEYWORDS: Data mining, Heart disease, Classification, Weka, c\#.net

\section{Abstracts of First National Congress of Medical Informatics, Mashhad, Iran, February 2017}

(C) 2017 The Authors. This is an open access article under the terms of the Creative Commons Attribution-NonCommercialNoDerivs License, which permits use and distribution in any medium, provided the original work is properly cited, the use is non-commercial and no modifications or adaptations are made. 